\title{
A RANDOMIZED ACTIVE CONTROLLED CLINICAL STUDY TO EVALUATE EFFICACY AND SAFETY OF RESVERATROL AS AN ADJUVANT THERAPY IN PATIENTS WITH HYPERTENSION
}

\author{
OJHA RAKESH ${ }^{1 *}$, KULKARNI PRANESH ${ }^{2}$, VYAS BHAVIN ${ }^{1}$
}

${ }^{1}$ Department of Pharmacology, Maliba College of Pharmacy, Uka Tarsadia University, Bardoli, Surat, Gujarat, India. ${ }^{2}$ Department of , Vikas Hospital, Rambaug, Kalyan (West), Maharashtra, India. Email: rakeshojha0712@gmail.com

Received: 09 October 2016, Revised and Accepted: 01 December 2016

\section{ABSTRACT}

Objectives: No long-term clinical efficacy and safety study of resveratrol as adjuvant therapy along with gold standard therapy has been conducted in patients with essential hypertension. The aim of this study was to investigate the efficacy and safety of resveratrol as an adjuvant therapy in newly diagnosed hypertensive patients.

Methods: In this randomized active-controlled study, hypertensive patients (male/female) of 20-65 years aged were randomized (1:1) to receive telmisartan $20 \mathrm{mg}$ or telmisartan $20 \mathrm{mg}$ plus resveratrol $1 \mathrm{~g}$ daily for 12 months. Efficacy variables included a change in systolic and diastolic blood pressure (DBP) from baseline and were followed up for every 3 months. Treatment-emergent adverse events were assessed.

Results: A total of 60 hypertensive patients were analyzed (telmisartan [n=30] and telmisartan plus resveratrol [n=30]). Resveratrol as an adjuvant with telmisartan significantly reduced in systolic $(\mathrm{p}<0.001)$ and DBP $(\mathrm{p}<0.001)$ as compared to telmisartan monotherapy. Change in systolic and DBP from baseline was significantly higher in telmisartan plus resveratrol group than telmisartan $(p<0.001)$ at all followed visits. Both the study drugs have similar safety profile and found well tolerable.

Conclusions: Resveratrol plus Telmisartan was found to be superior over telmisartan monotherapy in reducing systolic and DBP in newly diagnosed hypertensive patients. Both the study drugs were effective with comparable safety profile. Our study supports the long-term clinical efficacy and safety study of resveratrol along with gold standard therapy in essential hypertension.

Keywords: Essential hypertension, Resveratrol, Systolic and diastolic blood pressure, Telmisartan.

(c) 2017 The Authors. Published by Innovare Academic Sciences Pvt Ltd. This is an open access article under the CC BY license (http://creativecommons. org/licenses/by/4. 0/) DOI: http://dx.doi.org/10.22159/ajpcr.2017.v10i1.15603

\section{INTRODUCTION}

In India, hypertension affected around $25-30 \%$ in urban and $10-15 \%$ in rural subjects, according to the World Health Organization health statistics 2012, the prevalence of hypertension in India was 23.1\% in men and $22.6 \%$ in women in equal or more than 25 years age. The raised blood pressure (BP) was a high-risk condition that caused approximately $51 \%$ of death from strokes and $45 \%$ from coronary artery disease in India [1]. Prevalence and economic burden related to hypertension have been increasing and are projected to be continued to increase [2].

Hypertension is a risk factor for the development of atherosclerosis, although the mechanisms have not been well elucidated [3]. The most common side effect of currently available gold standard therapy (angiotensin-converting enzyme inhibitors and angiotensin II Type 1 (AT1) receptor antagonists) is a cough $(\sim 9 \%)$, angioedema $(\sim 3 / 1000)$, hypokalemia, and hyperkalemia. The most common side effects of betablockers and diuretics are depression, lower high-density lipoprotein, and raises triglycerides, hypoglycemia, rebound hypertension, glucose intolerance, increased uric acid, erectile dysfunction in men and doserelated hypokalemia [4,5]. To manage hypertension effectively, often combination therapy and adjusting dose of gold standard therapy from low to high is required to prevent mortality. This may result in longterm safety issues and reduce the financial burden to patients and their family. The agent that helps in controlling BP is the better options for management of hypertension. The combination treatment therapy may also slow down the natural disease progression and reduces the complications associated with hypertension. To prevent side effects and financial burden due to combination therapy and high dose of gold standard therapy, there is need of an effective adjuvant therapy which is free from side effects, cost-effective, and enhances the efficacy of current gold standard therapy without the need of switching to aggressive therapy. Furthermore, there is a need of adjuvant therapy which keeps us healthy and useful as prophylaxis for the patients with risk factor of cardiovascular disease.

Resveratrol is a stilbenoid type of natural phenol, and a phytoalexin produced naturally by several plants [6]. Resveratrol reduces oxidative stress, improves vascular function, attenuates high BP, and prevents cardiac hypertrophy [7]. Another pre-clinical study suggested that resveratrol prevents monocrotaline-induced pulmonary hypertension in rats. Resveratrol exerts anti-inflammatory, antioxidant, and antiproliferative effects in the pulmonary arteries, which may contribute to the prevention of pulmonary hypertension [8]. Resveratrol administration reduces metabolic disturbances and lowers $\mathrm{BP}$ in obese rats [9]. This indicates that the possible use of resveratrol in combination with current anti-hypertensive therapy as an adjuvant therapy would be effective in preventing and treating high BP. Shortterm clinical use of Resveratrol $1 \mathrm{~g} /$ daily (500 mg twice daily [BD]) was found to be effective in decreasing BP when compared to placebo after 45 days [10]. Another 4 week clinical study (in 10 subjects) suggested that there was no change in BP after administration of resveratrol (1 g/daily) [11].

We hypothesized that resveratrol could be one of the adjuvant therapy along with gold standard anti-hypertensive therapy, which may lead to significantly improve the effectiveness of gold standard therapy in the management of hypertension and also decrease the financial burden to the patients along with good safety profile. Moreover, no long- 
term clinical efficacy and safety study of resveratrol in hypertension patient have been conducted. Based on the above facts, the proposed clinical study designed to evaluate efficacy and safety of Resveratrol, as adjuvants in patients with recently diagnosed with hypertensive patients who were stable on telmisartan monotherapy.

\section{METHODS}

A prospective, randomized, parallel, open-label, comparative, active-controlled clinical study was conducted at the single study center in India. From September 2014 to August 2015, 60 patients of either sex of 20-65 years who had stage I hypertension (systolic BP [SBP] 140-159 mmHg and diastolic BP [DBP] 90-99 $\mathrm{mmHg}$ ), and stable on telmisartan $20 \mathrm{mg}$ monotherapy were enrolled. Pregnant women, lactating mothers, history of severe heart disease, hepatic disease and renal dysfunction, willing to use other antioxidant supplementation rather than resveratrol, grapes allergy, the patient was receiving drug which affect BP, and regular alcoholics were excluded from this study. During the screening visit on the day, medical history was obtained; physical examination and laboratory investigations were performed. Medications considered necessary for the patient and which does not interact with the study medication were allowed. All patients were explained the procedure clearly and written informed consent from each participant was obtained before their participation in the study. The protocol was approved by Safety, Health and welfare Ethics Committee, registered under Drug controller general of India (Reg No: ECR/632/Inst/MH/2014). The study was conducted in compliance with the Ethical principles of Declaration of Helsinki; Good Clinical Practices guidelines issued by the Central Drugs Standard Control Organization, Ministry of Health, Government of India; Ethical guidelines for biomedical research on human participants, Indian Council of Medical Research, New Delhi; and International conference on harmonization of technical requirements for registration of pharmaceuticals for human use - guideline for good clinical practices. The trial was registered with Clinical Trial Registry-India before initiation of the study. Enrolled patients were randomized in 1:1 in 2 groups as per the computer generated sheet. The patients randomized in the interventional group (test group) were received telmisartan $20 \mathrm{mg}$ once daily along with oral resveratrol $500 \mathrm{mg}$ twice a day (a total of $1 \mathrm{~g} /$ daily). The patients randomized to control group were received only telmisartan $20 \mathrm{mg}$ once daily. The treatment duration was 12 months for both the groups. Compliance to study drugs was assessed using pill count method at each assessment visit after dispending study drugs to patients.

Both systolic and DBP was assessed twice with 2 minutes apart after a 5-minutes rest in the sitting position, using an auscultatory method of measurement with a properly calibrated and validated mercury sphygmomanometer. Two measurements were made 2 minutes apart. SBP is the point at which the first of two or more sounds is heard (phase 1), and DBP is the point before the disappearance of sounds (phase 5). The mean of the two recordings was taken for analysis. Heart rate was measured for 1 minute in the 2-minute interval between $\mathrm{BP}$ measurements. Body temperature was measured by thermometer. $\mathrm{BP}$ (SBP and DBP) and heart rate were measured at baseline (before the start of study treatment), end of $3,6,9$, and 12 months in both the treatment groups.

\section{Statistical analysis}

Based on a power of $80 \%$ and a type I error rate of alpha $=0.05$ (two-tailed), a sample size of at least 52 patients per group was required to detect a clinically significant difference of $08 \mathrm{mmHg}$ in the change in BP (with standard deviation [SD] of 10.26 [12]) between both groups. Considering dropout rate of $15 \%$, total sample size will be approx. 60 patients ( 60 patients in each group). Categorical data were presented as absolute number/percentage of patients while quantitative data were presented as mean \pm SD. Depending on the distribution of data appropriate parametric or non-parametric test was used to find p value. Unpaired " $\mathrm{t}$ "/Man-Whitney test was used to analyze the quantitative data for between group comparisons. Within-group comparison was performed using paired $t$-test or Wilcoxan test for quantitative data based on the distribution of data. Missing data were handled using Mean substitution or Last observation carried forward method. Chi-square test/fisher exact test was used to compare the categorical or qualitative data of both the treatment groups. Normality tests (KS and SW test) were used to detect distribution of data for numerical data. $p<0.05$ was considered as the statistical significant difference.

\section{RESULTS}

There was no patient dropout from each group during the study period. At the end of the study, totally 30 patients in each group (control and intervention group) completed the study and subjected to statistical analysis. A consort diagram is presented showing the flow of participants through the study (Fig. 1)

Demographic and clinical characteristic of patients of both the treatment groups were comparable (Table 1).

At the baseline, there was no significant difference between both groups regarding age, gender, body weight, duration of disease and smoking, systolic and DBP. The prevalence of hypertension in the family was also comparable in both the treatment groups (Table 1).

Before the start of study drug treatment (at baseline), SBP and DBP were comparable in both the treatment group. There was gradual reduction in blood SBP and DBP over a period of 12 months hours in both the treatment groups as observed from the reduction trend in the SBP and DBP from baseline (Table 2).

In both the treatment groups, reduction in SBP and DBP was statistically significant when compared to baseline (within group comparison). Between-groups comparison showed that reduction in SBP and DBP was significant greater in test group at 3, 6, 9, and 12 months when compared to control ( $\mathrm{p}<0.005$ at each time point) (Table 2).

There was a significant improvement in SBP and DBP in both the treatment groups from baseline. However, improvement in SBP and DBP at 3, 6, 9, and 12 months from baseline was significantly greater in test group as compared to control group (Table 3).

The difference between the treatments for the change in SBP and DBP from baseline was statistically significant (Table 3 ).

\section{DISCUSSION}

In this prospective, randomized, parallel, open-label, comparative, active controlled, single center clinical study, resveratrol as adjuvant therapy significantly decrease SBP and DBP in patients with hypertension.

Table 1: Demographic and baseline clinical characteristics

\begin{tabular}{llll}
\hline Patient characteristic & $\begin{array}{l}\text { Control } \\
\mathbf{n = 3 0}\end{array}$ & Test (n=30) & $\mathbf{p}$ \\
\hline Demography & & & \\
$\quad$ Gender (male/female) & $21 / 9$ & $18 / 12$ & $>0.05$ \\
$\quad$ Age (year) & $39.7 \pm 3.6$ & $44.0 \pm 3.10$ & $>0.05$ \\
Height (Cm) & $167.1 \pm 5.3$ & $168.9 \pm 5.20$ & $>0.05$ \\
$\quad$ Weight (Kg) & $80.8 \pm 3.9$ & $78.5 \pm 4.62$ & $>0.05$ \\
Smoking & & & \\
$\quad$ Yes & 22 & 20 & $>0.05$ \\
$\quad$ No & 8 & 10 & $>0.05$ \\
$\quad$ Duration of disease (months) & $1.8 \pm 0.9$ & 1.70 .7 & \\
Family history of hypertension & & & \\
$\quad$ Yes & 24 & 23 & 0.05 \\
$\quad$ No & 6 & 7 & \\
\hline
\end{tabular}

Control=telmisartan $20 \mathrm{mg}$, test=telmisartan $20 \mathrm{mg}+$ resveratrol $500 \mathrm{mg}$ twice daily. Values are expressed as Mean \pm standard deviation except for gender, family history of dyslipidemia and smoking. Absolute values are expressed for gender, family history of dyslipidemia and smoking. $P$ value by unpaired $t$-test for continuous variables, fisher exact test for gender, family history of dyslipidemia and smoking 


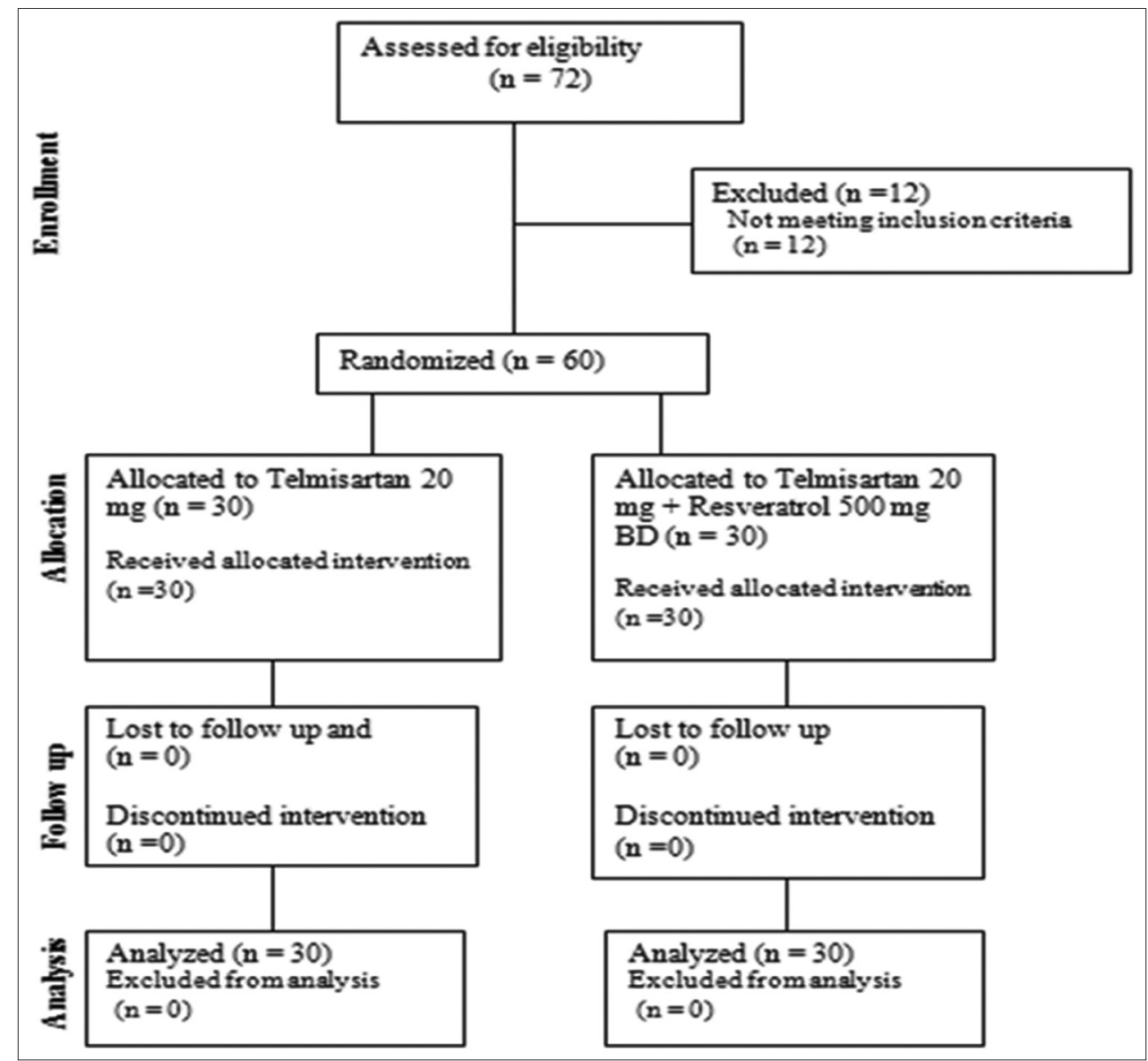

Fig. 1: Flow of participants through the study

Table 2: Blood pressure $(\mathrm{mmHg})$ at baseline and over the period of 12 months after study drug treatments

\begin{tabular}{llll}
\hline Parameters & Control $(\mathbf{n}=\mathbf{3 0})$ & Test $(\mathbf{n = 3 0})$ & $\mathbf{p}$ \\
\hline Baseline & & & \\
$\quad$ SBP & $151.7 \pm 8.3$ & $156.0 \pm 8.14$ & $>0.05$ \\
$\quad$ DBP & $100.3 \pm 8.5$ & $104.3 \pm 6.79$ & $>0.05$ \\
End of 3 months & & & \\
$\quad$ SBP & $135.0 \pm 5.1^{*}$ & $131.0 \pm 5.48^{*}$ & $<0.05$ \\
DBP & $84.7 \pm 5.7^{*}$ & $82.0 \pm 4.07^{*}$ & $<0.05$ \\
End of 6 months & & & \\
SBP & $127.7 \pm 7.3^{*}$ & $123.7 \pm 4.90^{*}$ & $<0.05$ \\
DBP & $83.7 \pm 4.9^{*}$ & $80.3 \pm 1.83^{*}$ & $<0.05$ \\
End of 9 months & & & \\
$\quad$ SBP & $123.7 \pm 4.9^{*}$ & $120.3 \pm 1.83^{*}$ & $<0.05$ \\
DBP & $81.7 \pm 3.8^{*}$ & $79.0 \pm 3.05^{*}$ & $<0.05$ \\
End of 12 months & & & \\
$\quad$ SBP & $123.0 \pm 4.7^{*}$ & $119.3 \pm 2.54^{*}$ & $<0.05$ \\
DBP & $81.0 \pm 3.1^{*}$ & $78.3 \pm 3.79^{*}$ & $<0.05$ \\
\hline
\end{tabular}

Values are expressed as mean \pm standard deviation. ${ }^{*} \mathrm{p}<0.05$ from baseline by paired $t$-test (within group comparison). Between-groups comparison was done using unpaired $t$-test, DBP: Diastolic blood pressure, SBP: Systolic blood pressure

Resveratrol as adjuvant therapy having acceptable safety profile and the most common adverse event is a gastrointestinal disturbance. Our study suggested that the addition of resveratrol to gold standard therapy of anti-hypertensive class of drug and significantly improve the efficacy of gold standard therapy, possibly due to synergistic action. The potential effect of resveratrol in the management of hypertension has been established in pre-clinical setting. In rodent model, resveratrol was effective in preventing obesity and oxidative stress and reducing the risk of hypertension and dyslipidemia in adult rats [10]. Resveratrol
Table 3: Change in blood pressure $(\mathrm{mmHg})$ from baseline to 3 months, 6, 9 and 12 after study drug treatments

\begin{tabular}{llll}
\hline Parameters & Control (n=30) & Test (n=30) & p \\
\hline End of 3 months & & & \\
$\quad$ SBP & $-16.7 \pm 8.8^{*}$ & $-25.0 \pm 78.61^{*}$ & $<0.05$ \\
DBP & $-15.7 \pm 7.3^{*}$ & $-22.3 \pm 7.74^{*}$ & $<0.05$ \\
End of 6 months & & & \\
$\quad$ SBP & $-24.0 \pm 10.7^{*}$ & $-32.3 \pm 9.71^{*}$ & $<0.05$ \\
DBP & $-16.7 \pm 6.6^{*}$ & $-24.0 \pm 7.70^{*}$ & $<0.05$ \\
End of 9 months & & & \\
SBP & $-28.0 \pm 8.9^{*}$ & $-35.7 \pm 8.58^{*}$ & $<0.05$ \\
DBP & $-18.7 \pm 7.3^{*}$ & $-25.3 \pm 7.76^{*}$ & $<0.05$ \\
End of 12 months & & & \\
SBP & $-28.7 \pm 9.7^{*}$ & $-36.7 \pm 7.58^{*}$ & $<0.05$ \\
DBP & $-19.3 \pm 8.7^{*}$ & $-26.0 \pm 7.70^{*}$ & $<0.05$ \\
\hline
\end{tabular}

Values are expressed as mean \pm standard deviation. ${ }^{*} \mathrm{p}<0.05$ from baseline by paired $t$-test (within group comparison). Between-groups comparison was done using unpaired $t$-test, DBP: Diastolic blood pressure, SBP: Systolic blood pressure

prevents monocrotaline-induced pulmonary hypertension in rats. Resveratrol exerts anti-inflammatory, antioxidant, and antiproliferative effects in the pulmonary arteries, which may contribute to the prevention of pulmonary hypertension [8]. Long-term resveratrol administration reduces metabolic disturbances and lowers BP in obese rats [9]. Chronic resveratrol enhances endothelium-dependent relaxation in aorta of spontaneously hypertensive rats [11]. Resveratrol has a preventive effect on hypertension-induced cardiac hypertrophy in rats [13]. Moreover, our study results were also consistent with the previous clinical reports, short-term clinical use of resveratrol $1 \mathrm{~g} /$ daily (500 mg BD) was found to be effective in decreasing BP when compared to Placebo after 45 days [12]. Another 4 week clinical study (in 10 
subjects) suggested that there was no change in BP after administration of resveratrol (1 g, $1.5 \mathrm{~g}$ and $2 \mathrm{~g}$ /daily) [14].

We also observed that resveratrol as adjuvant therapy was found to be effective in improving SBP and DBP in patients with hypertension. Results of our study showed the combination of resveratrol and gold standard therapy of anti-hypertensive class of drug significantly improve SBP and DBP levels as compared to the monotherapy; this was possibly due to synergistic action. Our study results with respect to anti-hypertensive effect of resveratrol were consistent with previous report $[12,14]$. Our study support the long-term clinical efficacy and safety study of resveratrol along with gold standard therapy in hypertension patients and suggested synergistic effect of resveratrol when given along with gold standard therapy as adjuvant therapy in a clinical setting in the management of hypertension.

\section{CONCLUSION}

Resveratrol as an adjuvant therapy was found to be well tolerated and effective in hypertension. Both the study drugs have comparable safety profile and found well tolerable. Resveratrol plus telmisartan was found to be superior over telmisartan monotherapy in reducing SBP and DBP in patients with essential hypertension.

\section{ACKNOWLEDGMENTS}

All authors would like to thank the subjects for their participation in this study.

\section{REFERENCES}

1. Prevalence of Hypertension High Among Lower, Middle Class Population in India. Available from: http://www.articles. timesofindia.indiatimes.com/2013-04-05/chandigarh/38305800 1 anti-hypertensive-medications-uncontrolled-hypertension-high-b̄ [Last accessed on 2016 Sep 20].

2. van de Vijver S, Akinyi H, Oti S, Olajide A, Agyemang C, Aboderin I, et al. Status report on hypertension in Africa - Consultative review for the $6^{\text {th }}$ Session of the African Union Conference of Ministers of Health on NCD's. Pan Afr Med J 2013;16:38.

3. Alexander RW. Theodore Cooper Memorial Lecture. Hypertension and the pathogenesis of atherosclerosis. Oxidative stress and the mediation of arterial inflammatory response: A new perspective. Hypertens 1995;25(2):155-61.

4. Chalmers J. Treatment guidelines in hypertension: Current limitations and future solutions. J Hypertens Suppl 1996;14(4):S3-8.

5. Tarazi RC, Fouad-Tarazi FM. Current therapy, present limitations and future goals for systemic hypertension. Am J Cardiol 1986;58(8):3D-7.

6. Mishra RN. Resveratrol-The new Rasayan (anti-aging) drug. Curr Res Med Med Sci 2011;1(1):5-18.

7. Dolinsky VW, Chakrabarti S, Pereira TJ, Oka T, Levasseur J, Beker D, et al. Resveratrol prevents hypertension and cardiac hypertrophy in hypertensive rats and mice. Biochim Biophys Acta 2013;1832(10):1723-33.

8. Csiszar A, Labinskyy N, Olson S, Pinto JT, Gupte S, Wu JM, et al. Resveratrol prevents monocrotaline-induced pulmonary hypertension in rats. Hypertension 2009;54(3):668-75.

9. Rivera L, Morón R, Zarzuelo A, Galisteo M. Long-term resveratrol administration reduces metabolic disturbances and lowers blood pressure in obese Zucker rats. Biochem Pharmacol 2009;77(6):1053-63.

10. Franco JG, Lisboa PC, Lima NS, Amaral TA, Peixoto-Silva N, Resende AC, et al. Resveratrol attenuates oxidative stress and prevents steatosis and hypertension in obese rats programmed by early weaning. J Nutr Biochem 2013;24(6):960-6.

11. Rush JW, Quadrilatero J, Levy AS, Ford RJ. Chronic resveratrol enhances endothelium-dependent relaxation but does not alter eNOS levels in aorta of spontaneously hypertensive rats. Exp Biol Med (Maywood) 2007;232(6):814-22.

12. Movahed A, Nabipour I, Lieben Louis X, Thandapilly SJ, Yu L, Kalantarhormozi $\mathrm{M}$, et al. Antihyperglycemic effects of short term resveratrol supplementation in Type 2 diabetic patients. Evid Based Complement Alternat Med 2013;2013:851267.

13. Liu Z, Song Y, Zhang X, Liu Z, Zhang W, Mao W, et al. Effects of trans-resveratrol on hypertension-induced cardiac hypertrophy using the partially nephrectomized rat model. Clin Exp Pharmacol Physiol 2005;32(12):1049-54.

14. Crandall JP, Oram V, Trandafirescu G, Reid M, Kishore P, Hawkins M, et al. Pilot study of resveratrol in older adults with impaired glucose tolerance. J Gerontol A Biol Sci Med Sci 2012;67(12):1307-12. 